\title{
2. Evans's Theorem on Abstract Riemann Surfaces with Null-Boundaries. II
}

\author{
By Zenjiro KURAMOCHI
}

Mathematical Institute, Osaka University

(Comm. by K. KUnUGI, M.J.A., Jan. 12, 1956)

Transfinite diameter. Let $A$ be an $m$-closed subset of $B$. We define the transfinite diameter of $A$ of order $n$ as follows

$$
\frac{1}{{ }_{A} D_{n}}=\frac{1}{2 \pi_{n} C_{2}}\left(\inf \sum_{\substack{p_{s}, p_{t} \in A \\ s<t, t, t=1}}^{n, n} G\left(p_{s}, p_{t}\right)\right)
$$

a) From the definition, it is clear, if $A_{1} \supseteq A_{2},{ }_{1} D_{n} \geqq{ }_{A_{2}} D_{n}$.

b) Let $\Omega$ be an ordinary neighbourhood containing $A$ with a compact relative boundary. Consider $1 / \Omega D_{n}=\frac{1}{2 \pi}\left(\frac{1}{{ }_{n} C_{2}} \inf _{p_{s}, p_{t} \in \Omega} G\left(p_{s}, p_{t}\right)\right)$. Then every $p_{s}$ is situated on $\partial \Omega$. $\sum_{s<t} G\left(p_{s}, p_{t}\right)=\sum_{\substack{i, j \neq s \\ i \neq j}} G\left(p_{i}, p_{j}\right)+\sum_{\substack{i \neq s \\ i \neq 1}}^{n} G\left(p_{s}, p_{i}\right)$. Then the sum of the first term does not depend on $p_{s}$ and $\sum_{i \neq s}^{n} G\left(p_{s}, p_{i}\right)=U\left(p_{s}\right)$ is a superharmonic function of $p_{s}$ for fixed $\left\{p_{i}\right\}$ in $\bar{R}$. We make $V_{M}\left(p_{i}\right)$ correspond to every point $p_{i}(i \neq s)$ such that $U\left(p_{s}\right) \geqq M$ in $\bigcup_{i} V_{M}\left(p_{i}\right)$, where $M \geqq$ $\max _{p_{s} \in \partial \Omega} U\left(p_{s}\right)$. Since $U\left(p_{s}\right)$ is $m$-lower semicontinuous, $U\left(p_{s}\right)$ attains its minimum $m^{*}$ at $z_{0}$ on an $\mathfrak{m}$-closed set $\Omega$. We show that $z_{0} \in \partial \Omega$. If it were not so, assume that $U\left(z_{0}\right)=m^{*} \leqq m=\min _{p_{s} \in \partial \Omega} U\left(p_{s}\right)$ in $\Omega$. Suppose $z_{0} \in B$, then by 3), $U\left(z_{0}\right)=\frac{1}{2 \pi} \int_{\partial V_{n}\left(z_{0}\right)} U(z) \frac{\partial G\left(z, z_{0}\right)}{\partial n} d s$, where $n$ is so large enough that $V_{n}\left(z_{0}\right) \subset \Omega$. Then there exists at least one point $r(\in R)$ such that $U(r) \leqq m^{*} \leqq m . \quad r$ must be in $\Omega-\bigcup V_{m}\left(p_{i}\right)$. But since $U\left(p_{s}\right)$ is harmonic non constant in $\Omega-\bigcup_{i} V_{M}\left(p_{i}\right)$ and $R$ is a null-boundary Riemann surface, $U\left(p_{s}\right)$ attains its minimum on $\partial \Omega$, by the minimum principle. Thus $U\left(z_{0}\right)>m$ in $\Omega$. This is absurd, therefore every $p_{i}$ is on $\partial \Omega$.

Let $\omega_{\Omega}(z)$ be the harmonic measure of $\Omega$ with respect to the domain $R-R_{0}-\Omega$ i.e. $\omega_{\Omega}(z)$ is harmonic in $R-\Omega-R_{0}$ and $\omega_{\Omega}(z)=0$ on $\partial R_{0}, \omega_{\Omega}(z)=1$ on $\partial \Omega$.

Since every $p_{\imath}$ is on $\partial \Omega$, the following can be proved as in euclidean space,

$$
\lim _{n=\infty} \frac{1}{\Omega} D_{n}=2 \pi / \int_{\partial \Omega} \frac{\partial \omega_{\Omega}(z)}{\partial n} d s=W_{\Omega}
$$


Let $\Omega_{k}(k=1,2,3, \cdots)$ be a decreasing sequence such that $\bigcap^{\infty} \Omega_{k}=B \supset A$. Since $R$ has a null-boundary, $\lim _{k=\infty} W_{\Omega_{k}}=\infty$ and since $A \subset \Omega_{k}$ for every $k$, we have

$$
\infty=1 /{ }_{A} D=\lim _{n} \frac{1}{2 \pi} \min \left(\sum G\left(p_{s}, p_{t}\right) /{ }_{n} C_{2}\right) .
$$

Suppose $n$ points $p_{1}, p_{2}, \cdots, p_{n}$ on $A$, we can choose an $(n+1)$ st point $p$ on $A$ so that

$$
V(p)=\left(\sum_{i=1}^{n} G\left(p, p_{\imath}\right)\right) / 2 \pi n \quad \text { is minimum, }
$$

because the above function is $\mathfrak{m}$-lower semicontinuous on $A$. Let ${ }_{A} V_{n}$ be the least upper bound of the minimum above defined as $p$, $p_{1}, \cdots, p_{n}$ vary on $A$. Then there exists a system $p_{1}^{*}, p_{2}^{*}, \cdots, p_{n}^{*}$ such that

$$
V\left(p, p_{1}^{*}, p_{2}^{*}, \cdots, p_{n}^{*}\right) \geqq{ }_{A} V_{n}-\frac{1}{n} \text { on } A .
$$

We denote by $V(p)$ the potential

$$
V(p)=\frac{1}{2 \pi n}\left(\sum_{i=1}^{n} G\left(p, p_{i}^{*}\right)\right) .
$$

This is the potential of a certain distribution of equal point mass on $A$ of total mass unity and it is clear that $V(p) \geqq{ }_{A} V_{n}-\frac{1}{2 \pi n}$ for all points on $A$ admitting $\infty$ as a possible value of either member. Furthermore, since $V(p)$ is lower semicontinuous, we can find a neighbourhood $v_{n}(q)$ for any point $q$ of $A$ such that $V(z) \geqq V(q)-\delta$ in $V_{n}(q)$, for any given number $\delta$, whence $\lim _{z_{i} \rightarrow q \in A} V\left(z_{i}\right) \geqq{ }_{A} V_{n}-\frac{1}{2 \pi n}$ for every sequence $\left\{z_{i}\right\}$ tending to $A$ in $\mathfrak{M}$-sense.

Since $G\left(p_{i}, p_{j}\right)=G\left(p_{j}, p_{i}\right)$.

$$
\left(\begin{array}{c}
n+1 \\
2
\end{array}\right) /{ }_{A} D_{n+1}=\frac{1}{2 \pi} \min _{p_{i} \in A}\left({ }_{i<k}^{1,2, \cdots, n+1} G\left(p_{i}, p_{k}\right)=\frac{1}{2} \frac{1}{2 \pi} \sum_{k=1}^{n+1} \sum_{\substack{j=1 \\
k \neq j}}^{n+1} G\left(p_{i}, p_{k}\right)\right) .
$$

Hence ${ }_{A} V_{n} \geqq 1 /{ }_{A} D_{n+1}$, therefore $V(M) \geqq 1 /{ }_{A} D_{n+1}-\frac{1}{2 \pi n}$.

Theorem 2. Let $A$ be an m-closed subset of $B$ of a null-boundary Riemann surface with a compact relative boundary $\partial R_{0}$. Then there exists a potential $U(z)$ such that 1) $U(z)$ is harmonic in $R-R_{0}$. 2) $U(z)=0$ on $\partial R_{0}$. 3) $\int_{\partial R_{0}} \frac{\partial U(z)}{\partial n} d s=2 \pi$. 4) $\lim _{z \rightarrow A} U(z)=\infty$.

Proof. Let $N(\geqq 3)$ be an integer. Then since $\lim _{n} 1 /_{A} D_{n+1}=\infty$, there exist, for any positive integer $m, n_{0}(N, m)$ number of points $p_{1}, p_{2}, \cdots, p_{n_{0}}$ such that

$$
\lim _{z \rightarrow A}^{\mathfrak{M}} V^{m}(z)=\lim _{z \rightarrow A} \frac{1}{2 \pi}\left(\sum_{i}^{n_{0}} G\left(z, p_{i}\right)\right) \geqq N^{m} .
$$


No. 1] Evans's Theorem on Abstract Riemann Surfaces with Null-Boundaries. II 9

Put $\sum_{m=1}^{\infty} V^{m}(z) / 2^{m}=U(z)$. Then we see easily that $U(z)$ is the function required.

$U(z)$ obtained above does not necessarily satisfy the condition

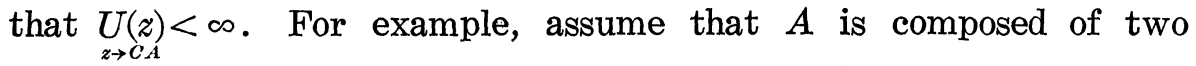
Martin's minimal points ${ }^{1)} p_{1}$ and $p_{2}$ on the same boundary component of harmonic dimension two $\mathrm{o}^{2)}$ and let $p_{3}$ be another non minimal point such that $G\left(z, p_{3}\right)=\frac{1}{2}\left(G\left(z, p_{1}\right)+G\left(z, p_{2}\right)\right)$. Then clearly $\delta\left(p_{3}, A\right)>0$ and $U(z)$ of the theorem must be a linear form of $G\left(z, p_{1}\right)$ and $G\left(z, p_{2}\right)$ and $U(z)$ satisfies the conditions above but not the last condition above-mentioned.

Let $\left\{R_{n}\right\}$ be an exhaustion with compact relative boundaries $\left\{\partial R_{n}\right\}$. Then $R-R_{n}$ is composed of a finite number of disjoint non compact surfaces $\left\{G_{n}^{i}\right\}(i=1,2, \cdots, i(n))$. Let $\left\{G_{n}^{i}\right\}$ be a sequence such that $G_{n}^{i} \supset G_{n+1}^{i^{\prime}}(n=1,2, \cdots)$ and $\bigcap_{n} G_{n}^{i}=0$. The equivalency of $\left\{G_{n}^{i}\right\}$ and $\left\{G_{n^{\prime}}^{i^{\prime}}\right\}$ is defined as usual. Let an ideal point correspond to an equivalent sequence and denote the set of all ideal boundary points by $\underline{B}$ and let $\underline{A}$ be a closed subset of $\underline{B}$ and let $A$ be the set of Martin's point on $\underline{A}$. Then $A$ is also closed in Martin's sense. Hence we have

Theorem 3. Let $A$ be the subset of $B$ on a closed subset $\underline{A}$ of B. Then there exists a harmonic function $U(z)$ satisfying the same conditions of Theorem 3 and moreover 5) $\lim _{\left.z \rightarrow p_{i} \in C A\right)} U(z)<\infty$.

In fact, let $q$ be a point of $C A$. Then there exists an ordinary neighbourhood $\mathfrak{B}(q)$ with a compact relative boundary such that $\mathfrak{B}(q) \cap A=0$. Then $\max _{z \in \partial \mathfrak{B}(q)} U(z) \leqq M$, whence $\lim _{z \in \mathfrak{B}(q)} U(z) \leqq M$ by maximum principle, because $U(z)$ has no mass in $\mathfrak{B}(q)$.

Set $A=B$. Then we have the following

Theorem 4. $R$ is a null-boundary Riemann surface, if and only if, there exists a harmonic function $U(z)$ with one negative logarithmic singularity at a point of $R$ which has limit co when z tends to $B$.

Because $R$ is clearly a null-boundary Riemann surface when the function mentioned above exists and it is easy to construct the function in this theorem from the function in Theorem 3.

Many other applications, for instance, to Nevanlinna's first and second foundamental theorems or to the Gross's property, will be omitted here.

1) If a positive harmonic function $U(z)$ has no smaller positive harmonic functions than itself except its own submultiple, $U(z)$ is called a minimal function. Martin proved that every positive harmonic function is represented uniquely by a linear form of minimal functions.

2) The number of linearly independent positive harmonic function $U_{i}(z)$ such that $U_{i}(z)$ is harmonic and $U_{i}(z)<\infty$ in $\bar{R}$ except $p$, is called the harmonic dimension of the component $p$. 